\title{
New Approaches to Anti-infective Treatment of Ventilator-Associated Respiratory Infections caused by Pseudomonas aeruginosa
}

Sebastian O Decker ${ }^{1}$, Ricardo Riebold da Costa1, Karsten Schmidt', Florian Uhle1, Christoph Lichtenstern'1, Thomas Bruckner², Dominic Störzinger ${ }^{3}$, Alexandra Heininger ${ }^{4}$, Alexis Ulrich ${ }^{5}$, Markus A Weigand ${ }^{1}$, Stefan Hofer ${ }^{6}$ and Thorsten Brenner ${ }^{1 *}$

${ }^{1}$ Department of Anesthesiology, Heidelberg University Hospital, Im Neuenheimer Feld, Heidelberg, Germany

${ }^{2}$ Institute of Medical Biometry and Informatics, University of Heidelberg, Im Neuenheimer Feld, Heidelberg, Germany

${ }^{3}$ Department of Pharmacy, Heidelberg University Hospital, Im Neuenheimer Feld, Heidelberg, Germany

${ }^{4}$ Department of Infectious Diseases, Heidelberg University Hospital, Im Neuenheimer Feld, Heidelberg, Germany

${ }^{5}$ Department of General, Visceral and Transplantation Surgery, Heidelberg University Hospital, Im Neuenheimer Feld, Heidelberg, Germany

${ }^{6}$ Department of Anesthesia, Westpfalzklinikum Kaiserslautern, Hellmut-Hartert-Straße, Kaiserslautern, Germany

\begin{abstract}
Study background: The purpose of this study was to evaluate recent strategies for anti-infective treatment in patients suffering from ventilator-associated respiratory infections caused by $P$. aeruginosa and to assess the riskbenefit profile of inhaled tobramycin.

Methods: Electronic health records of patients suffering from respiratory infections caused by $P$. aeruginosa from 2011-2014 were retrospectively screened.

Results: In 81 patients, $P$. aeruginosa was found in respiratory secretions, of which 26 patients suffered from ventilator-associated pneumonia (VAP), whereas 55 patients only fulfilled criteria of ventilator-associated tracheitis (VAT). Inhaled tobramycin was used in 14 patients with VAP and 31 patients with VAT. In this context, inhaled tobramycin was shown to be safe (e.g. no bronchoconstriction or systemic toxicity) and did not result in an increase of tobramycin resistant strains in respiratory secretions at 90 days following study inclusion. However, a clear clinical benefit (e.g. reduced need for i.v. antibiotics) could not be shown.

Conclusion: Inhaled tobramycin was shown to be a suitable approach with a favorable risk profile to treat patients suffering from respiratory infections (VAP, VAT) caused by $P$. aeruginosa. The clinical benefit needs to be reevaluated in a larger prospective investigation.
\end{abstract}

Keywords: Ventilator-associated pneumonia; Ventilator-associated tracheitis; P. aeruginosa; Inhaled tobramycin; Multidrug resistance

\section{Introduction}

Hospital-acquired pneumonia (HAP) is one of the most common types of nosocomial infection [1-3]. It has a considerable economic impact since its occurrence is associated with a prolonged hospital stay $[1,2]$. Ventilator-associated pneumonia (VAP) is a subgroup of HAP that affects patients on mechanical ventilation, who have a six- to twenty-fold increased risk of developing pneumonia [4,5]. VAP is associated with a need for prolonged mechanical ventilation, prolonged intensive care unit (ICU) stay, as well as increased mortality [6]. In order to reduce the incidence and to optimize the outcome of VAP, special ventilator care bundles have been established [7]. Moreover, an adequate antibiotic treatment regime is a prerequisite for a successful therapy of VAP, which is associated with several problems (e.g. multidrug resistant strains). Physicians are therefore faced with a high risk of making the wrong antibiotic choice due to increasing rates of multidrug resistant strains (e.g. P. aeruginosa) [7-9]. Besides, many antibiotic drugs reach inadequate drug levels in the epithelial lining fluid. Within this context, inhaled antibiotics have been shown to be advantageous [10-13]. Furthermore, the use of inhaled antibiotics might be able to reduce the use of intravenous antibiotics, resulting in fewer drug interactions and undesirable side effects (e.g. nephrotoxicity). Nevertheless, the potential risks of inhaled antibiotics also need to be taken into account (e.g. the development of resistance, undesirable side effects like bronchoconstriction or coughing attacks, and insufficient antibiotic concentrations in unventilated alveoli). Although this approach has been well-examined in patients with cystic fibrosis [14], the evidence for the use of inhaled antibiotics in critically ill patients suffering from VAP with $P$. aeruginosa is restricted $[15,16]$.
Accordingly, recent guidelines only give a weak recommendation for the additional use of inhaled antibiotics in high risk patients suffering from VAP due to gram-negative bacilli. However, medical societies (e.g. the European Society of Clinical Microbiology and Infectious Diseases (ESCMID)) reclaim an urgent need for additional investigations, assessing the use of nebulized antibiotics for the treatment of pneumonia due to multidrug resistant pathogens [17-19].

Ventilator associated tracheitis (VAT) is supposed to be a precursor of VAP [20], representing an infection of the lower respiratory tract involving the conduction zones, but without affecting the gas exchanges zones. Therefore VAT is considered to be a separate clinical entity [21] Since clinical data from well-designed studies are rare [21], recent guidelines cannot recommend an antibiotic treatment of VAT [18]. However, due to the growing importance of multidrug resistant strains in the pathogenesis of VAT and/or VAP, the ESCMID considers the

*Corresponding author: Thorsten Brenner, Department of Anesthesiology, Heidelberg University Hospital, Im Neuenheimer Feld 110, 69120 Heidelberg, Germany, Tel: +496221 56 39418; Fax: +49 6221565345 Email: thorsten.brenner@med.uni-heidelberg.de

Received January 05, 2018; Accepted January 24, 2018; Published January 27 2018

Citation: Decker SO, Riebold da Costa R, Schmidt K, Uhle F, Lichtenstern C et al. (2018) New Approaches to Anti-infective Treatment of Ventilator-Associated Respiratory Infections caused by Pseudomonas aeruginosa. J Med Microb Diagn 7: 270. doi:10.4172/2161-0703.1000270

Copyright: ( 2018 Decker SO, et al. This is an open-access article distributed under the terms of the Creative Commons Attribution License, which permits unrestricted use, distribution, and reproduction in any medium, provided the original author and source are credited. 
use of nebulized antibiotics. In parallel, the ESCMID warrants further studies, in order to assess the clinical benefit of inhaled tobramycin in cases of respiratory infections [19].

Therefore, this clinical study was performed in order to retrospectively assess recent strategies for the anti-infective treatment of patients suffering from respiratory infections (such as VAP or VAT) caused by $P$. aeruginosa and to evaluate the risk-benefit profile of inhaled tobramycin.

\section{Material and Methods}

This retrospective, observational clinical study was approved by the Ethics Committee of the Medical Faculty of Heidelberg, Germany (Trial-Code-Nr. S-094/2015). The database of the Department of Infectious Disease, Medical Microbiology and Hygiene of the Heidelberg University Hospital, Germany was screened for the presence of $P$. aeruginosa in respiratory secretions of each subject treated in the surgical ICU of the Heidelberg University Hospital, Germany between 2011 and 2014. VAP and VAT were diagnosed according to the criteria of the Centers for Disease Control and Prevention (CDC) [22] (Electronic Supplementary Materials 1, 2). Patients were evaluated from the day of $P$. aeruginosa detection in respiratory secretions and were reevaluated for VAP or VAT 1, 2, 7, 14, 21 and 28 days afterwards. The resistance pattern of each $P$. aeruginosa strain was further described based on the definition of multidrug resistant gram-negative bacteria (MRGN) according to an expert panel of the German Society for Hygiene and Microbiology (Electronic Supplementary Material 3). Apart from P. aeruginosa, further relevant microbiological findings in respiratory secretions, ensuing antibiotic treatment regimens as well as changes in the microbiological resistance profiles were also recognized within the 28 -day observation period. In order to assess the incidence of tobramycin resistances following the use of inhaled tobramycin, a long-term follow-up was performed at 90 days following study inclusion. Patient characteristics included age, gender, previous operations, medical history, chronic lung diseases (CLD), cardiac insufficiency (NYHA I-IV), renal insufficiency (with creatinine $>1.2 \mathrm{mg} / \mathrm{dl}$ ), coronary heart disease, immunosuppressive state, post-transplantation state, cancer and former radio- or chemotherapy, the Acute Physiology, Chronic Health Evaluation IIScore (APACHE II), the Sepsis-related Organ Failure Assessment score (SOFA), the Simplified Acute Physiology Score (SAPS) and the Clinical Pulmonary Infection Score (CPIS). Outcome parameters were defined as follows: length of ICU stay, duration of mechanical ventilation, tracheotomy rate, length of hospital stay (date of ICU admission to hospital discharge or death) and disappearance or persistence of $P$. aeruginosa. Based on local resistance patterns, the intravenous (i.v.) antibiotic drugs piperacillin/tazobactam, ciprofloxacin, imipenem/ cilastatin, meropenem, ceftazidime, colistin and tobramycin were classified as being effective against $P$. aeruginosa. In patients suffering from VAP, antibiotic therapy was defined as empiric from the start of antibiotic therapy due to the (clinical and radiological) manifestation of VAP up to the first occurrence of $P$. aeruginosa in respiratory tract secretions with knowledge of the respective resistance pattern. From then on, antibiotic treatment was defined as targeted. The same holds true for patients presenting with VAT, although antibiotic therapy was most frequently due to a simultaneously existing abdominal focus. The cumulative duration of use of these antibiotics in each patient was calculated by adding up the days a patient received at least one of these antibiotic drugs effective against $P$. aeruginosa. In patients receiving more than one antibiotic drug, the cumulative duration of use was calculated for each single antibiotic drug and then added up. Inhaled tobramycin was applied using an air-driven nebulizer via the artificial airway at a dosage of $300 \mathrm{mg}$ twice a day.

The resulting study data were entered into an electronic database (Microsoft Excel 2011, Microsoft Corp., Redmond, WA) and evaluated using SSPS Version 22.0 (Statistical Product and Services Solutions, SPSS Inc., Chicago, IL, USA). Data were checked for normal distribution by the Kolmogorov-Smirnov test. Due to non-normally distributed data, non-parametric tests were used for the statistical analyses (the Mann-Whitney test for quantitative data and the chisquared test for categorical data). To compare different subgroups of patients, the Kruskal-Wallis test was performed. Data are presented as median with interquartile range (IQR) or absolute and relative frequencies, depending on the underlying distribution. A p-value $<0.05$ was considered statistically significant.

\section{Results}

Within the four-year observation period, a positive culture for $P$. aeruginosa from respiratory tract secretions was obtained in 89 patients. Due to the absence of basic data, eight patients had to be excluded, such that 81 patients were screened for the presence of VAP or VAT (according to the CDC criteria as described above (Electronic Supplementary Materials 1 and 2). In total, 26 patients (32.1\%) with a positive culture for $P$. aeruginosa were found to suffer from VAP, whereas $55(67.9 \%)$ patients had to be classified as suffering from VAT.

\section{Subgroup of patients with VAP $(n=26)$}

Patients suffering from VAP $(\mathrm{n}=26)$ caused by $P$. aeruginosa were found to be hospitalized for nearly one month (27 d (13-34d)) and were in need for ICU treatment for more than three weeks (23 d (9-32 d)) prior to VAP onset. Initial ICU admission was due to a septic disease state in all cases. Median mechanical ventilation time was six days (144 h (32-662 h) until VAP occurred and patients were found to be critically ill as assessed by different disease severity scores. Concerning known illnesses, more than $30 \%$ of patients with VAP were found to suffer from chronic obstructive lung disease, which is known to be a risk factor for an infection with $P$. aeruginosa. Due to the oncologic focus of our surgical ICU, two-thirds of patients with VAP were found to suffer from an oncologic disease. Further relevant demographic and baseline clinical data of VAP patients are presented in detail in Table 1.

Depending on the initial empiric antibiotic treatment regime, patients suffering from VAP $(n=26)$ could be divided into five subgroups: empiric monotherapy with i.v. antibiotics was performed in $46 \%(n=12)$ of patients (Group I), whereas $4 \%(n=1)$ of patients were treated with tobramycin p.i. as monotherapy (Group II). Empiric i.v. monotherapy in combination with tobramycin p.i. was performed in $4 \%(n=1)$ of patients (Group III), whereas $35 \%(n=9)$ of patients were treated with at least two i.v. antibiotics (plus inhaled tobramycin in two of these patients) (Group IV). In $12 \%(n=3)$ of patients, no empiric therapy was performed (Group V). The different subgroups were comparable concerning their demographic and baseline clinical data. Moreover, clinical outcome measures (28-day mortality, 90day mortality, length of ICU stay, length of hospital stay, duration of mechanical ventilation, etc.) did not differ significantly between the five subgroups (data not shown). As described above, inhaled tobramycin was empirically applied to $16 \%(n=4)$ of VAP patients. Subsequent targeted antibiotic treatment strategies are also presented in Figure 1 and resulted from individual resistance patterns as well as an assessment of the recent clinical condition. In terms of targeted therapy, inhaled tobramycin was added in $38 \%(\mathrm{n}=10)$ of patients and was therefore applied to $54 \%(n=14)$ of all VAP patients. 
Citation: Decker SO, Riebold da Costa R, Schmidt K, Uhle F, Lichtenstern C, et al. (2018) New Approaches to Anti-infective Treatment of VentilatorAssociated Respiratory Infections caused by Pseudomonas aeruginosa. J Med Microb Diagn 7: 270. doi:10.4172/2161-0703.1000270

Page 3 of 9

\begin{tabular}{|c|c|c|c|c|}
\hline Variables & VAP patients $(n=26)$ & Without tobramycin p.i. $(n=12)$ & With tobramycin p.i. $(n=14)$ & p-value \\
\hline \multicolumn{5}{|c|}{ Demographic data } \\
\hline Gender male [n] & $21(80.7 \%)$ & $9(75 \%)$ & $12(85.7 \%)$ & 0.422 \\
\hline Age (years) & $67(55-72)$ & $62(50-70)$ & $69(63-73)$ & 0.060 \\
\hline \multicolumn{5}{|c|}{ Known illnesses } \\
\hline Diabetes mellitus & $10(38.5 \%)$ & $4(33.3 \%)$ & $6(42.9 \%)$ & 0.464 \\
\hline Arterial hypertension & $17(65.4 \%)$ & $6(50 \%)$ & $11(78.6 \%)$ & 0.133 \\
\hline Coronary heart disease & $7(26.9 \%)$ & $3(25 \%)$ & $4(28.6 \%)$ & 0.596 \\
\hline Chronic obstructive lung disease & $8(30.8 \%)$ & $4(33.3 \%)$ & $4(28.6 \%)$ & 0.563 \\
\hline Renal insufficiency & $7(26.9 \%)$ & $3(25 \%)$ & $4(28.6 \%)$ & 0.596 \\
\hline Hepatic insufficiency & $10(38.5 \%)$ & $5(41.7 \%)$ & $5(35.7 \%)$ & 0.536 \\
\hline Oncologic disease & $16(61.5 \%)$ & $7(58.3 \%)$ & $9(64.3 \%)$ & 0.536 \\
\hline Transplantation & $2(7.7 \%)$ & $1(8.3 \%)$ & $1(7.1 \%)$ & 0.720 \\
\hline \multicolumn{5}{|c|}{ Baseline clinical data* $^{*}$} \\
\hline Hospital length of stay [d] & $27(13-34)$ & $17(9-38)$ & $29(23-34)$ & 0.595 \\
\hline ICU length of stay [d] & $23(9-32)$ & $14(8-32)$ & $27(17-32)$ & 0.595 \\
\hline Mechanical ventilation $[\mathrm{h}]$ & $144(32-662)$ & $144(40-428)$ & $186(32-677)$ & 0.742 \\
\hline APACHE II & $13(11-16)$ & $12(10-16)$ & $14(11-16)$ & 0.432 \\
\hline SOFA & $8(4-14)$ & $8(4-14)$ & $8(5-11)$ & 0.781 \\
\hline SAPS & $49(40-59)$ & $49(36-64)$ & $49(42-55)$ & 0.940 \\
\hline $\mathrm{paO} 2 / \mathrm{FiO}_{2}$ & $240(190-278)$ & $240(200-270)$ & $240(188-275)$ & 0.898 \\
\hline \multicolumn{5}{|c|}{ Baseline microbiological data ${ }^{*}$} \\
\hline Pseudomonas 3-MRGN & $3(11.5 \%)$ & $1(8.3 \%)$ & $2(14.3 \%)$ & 0.560 \\
\hline Pseudomonas 4-MRGN & $0(0 \%)$ & $0(0 \%)$ & $0(0 \%)$ & - \\
\hline Tobramycin-resistance & $0(0 \%)$ & $0(0 \%)$ & $0(0 \%)$ & - \\
\hline
\end{tabular}

Legends: APACHE II: Acute Physiology and Chronic Health Evaluation II - Score; SOFA: Sepsis-related Organ Failure Assessment score; SAPS: Simplified Acute Physiology Score; 3-MRGN: Multi-Resistant Gram-Negative Bacteria with Resistance Against Three Main Antibiotic Drug Classes; 4-MRGN: Multi-Resistant GramNegative Bacteria with Resistance against Four Main Antibiotic Drug Classes; * Data expressed by number (percentage) or median with accompanying quartiles (Q1-Q3).

Table 1: Demographic and baseline clinical data of patients suffering from VAP $(n=26)$.

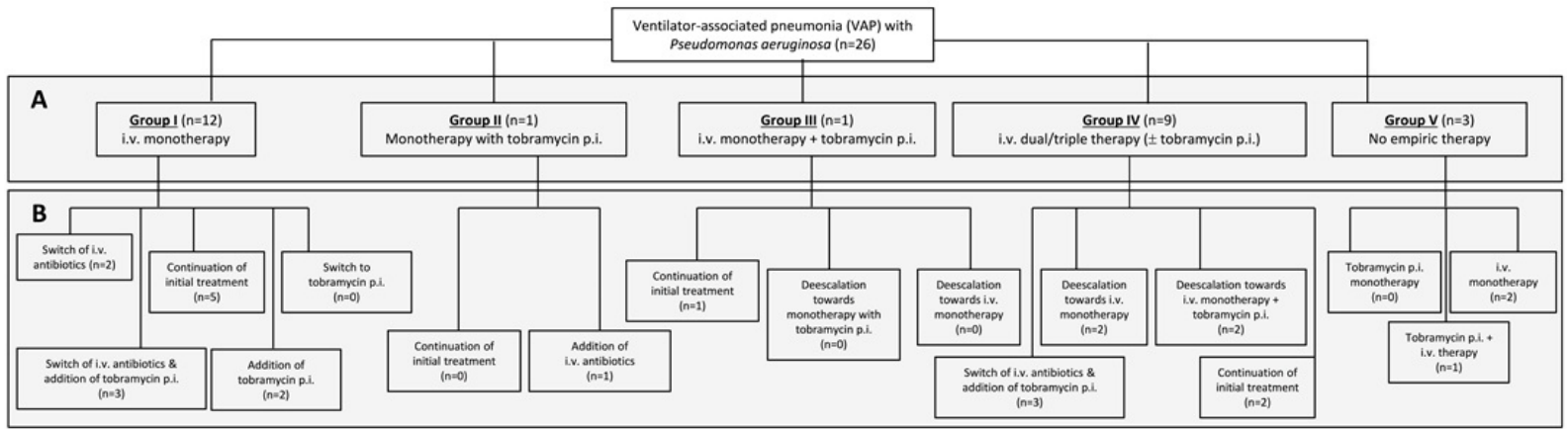

Figure 1: Empiric and targeted antibiotic treatment regimes in patients suffering from ventilator-associated pneumonia (VAP) with Pseudomonas aeruginosa. (A) Initial empiric choice of antibiotics and (B) subsequent targeted antibiotic treatment regimes in patients suffering from VAP caused by Pseudomonas aeruginosa.

Patients with and without inhaled tobramycin were comparable concerning their baseline characteristics (Table 1). Concerning tobramycin-related side effects, none of the participating patients suffered from bronchoconstrictive episodes or coughing attacks during the inhalation procedure. Moreover, the incidence of acute renal failure (ARF) as well as the need for renal replacement therapy (such as signs for systemic nephrotoxicity induced by aminoglycosides) did not differ significantly between patients with and without inhaled tobramycin. The incidence of other organ failures (e.g. acute liver failure (ALF) or acute respiratory distress syndrome (ARDS)) as well as several disease severity scores (APACHE II, SAPS \& SOFA) were also comparable between these two subgroups of patients within the 28-day observation period. No significant differences appeared concerning the Clinical Pulmonary Infection Score (CPIS) (Table 2). Mortality, length of hospital and length of ICU stay were found to be comparable between the two subgroups. However, the total duration of mechanical ventilation as well as the need for tracheostomy was shown to be significantly increased in patients treated with inhaled tobramycin (Table 2). This might be due to the fact that inhaled tobramycin was mostly used as a rescue therapy in cases of recurrent and/or persistent pulmonary infections caused by $P$. aeruginosa. Accordingly, the duration of mechanical ventilation up to the start of treatment with inhaled tobramycin was $364 \mathrm{~h}(33-677 \mathrm{~h})$, which exceeded the median duration of mechanical ventilation in VAP patients not treated with inhaled tobramycin $(85 \mathrm{~h}(9-286 \mathrm{~h}))$. Therefore, inhaled tobramycin was not apparently responsible for the prolonged need for mechanical ventilation (with subsequently higher rates of tracheostomy) in VAP patients, but rather represents a therapeutic approach to overcome a delayed response to conventional treatment with intravenously administered antibiotics. 
Citation: Decker SO, Riebold da Costa R, Schmidt K, Uhle F, Lichtenstern C, et al. (2018) New Approaches to Anti-infective Treatment of VentilatorAssociated Respiratory Infections caused by Pseudomonas aeruginosa. J Med Microb Diagn 7: 270. doi:10.4172/2161-0703.1000270

Page 4 of 9

\begin{tabular}{|c|c|c|c|c|}
\hline Variables & VAP patients $(n=26)$ & without tobramycin p.i. (n=12) & with tobramycin p.i. $(n=14)$ & p-value \\
\hline \multicolumn{5}{|c|}{ Organ failures up to 28 days } \\
\hline ARF & $14(53.8 \%)$ & $6(50.0 \%)$ & $8(57.1 \%)$ & 0.512 \\
\hline ALF & $21(80.8 \%)$ & $11(91.7 \%)$ & $10(71.4 \%)$ & 0.213 \\
\hline ARDS & $7(26.9 \%)$ & $3(25.0 \%)$ & $4(28.6 \%)$ & 0.596 \\
\hline \multicolumn{5}{|c|}{ Disease severity scores up to 28 days* } \\
\hline APACHE II & $13.6(10.9-15.8)$ & $12.7(10.3-14.2)$ & $14.2(11.6-15.8)$ & 0.231 \\
\hline SOFA & $7.6(3.5-13.7)$ & $7.5(2.9-13)$ & $6.8(4.4-10.5)$ & 1.000 \\
\hline SAPS & $49.1(33.7-58.3)$ & $38.2(23.9-50.1)$ & $49.3(45.3-58.3)$ & 0.403 \\
\hline CPIS & $4(3.5-5)$ & $4.2(3-5)$ & $4(3.8-4.6)$ & 0.940 \\
\hline \multicolumn{5}{|c|}{ Microbiological follow-up data within 28 days } \\
\hline Pseudomonas 3-MRGN & $3(11.5 \%)$ & $1(8.3 \%)$ & $2(14.3 \%)$ & 0.359 \\
\hline Pseudomonas 4-MRGN & $1(3.8 \%)$ & $0(0 \%)$ & $1(7.1 \%)$ & 0.462 \\
\hline Tobramycin-resistance & $1(3.8 \%)$ & $0(0 \%)$ & $1(7.1 \%)$ & 0.462 \\
\hline \multicolumn{5}{|c|}{ Microbiological follow-up data within 90 days } \\
\hline Pseudomonas 3-MRGN & $0(0 \%)$ & $0(0 \%)$ & $0(0 \%)$ & --- \\
\hline Pseudomonas 4-MRGN & $1(3.8 \%)$ & $1(8.3 \%)$ & $0(0 \%)$ & 0.500 \\
\hline Tobramycin-resistance & $0(0 \%)$ & $0(0 \%)$ & $0(0 \%)$ & -.-- \\
\hline \multicolumn{5}{|c|}{ Antibiotic stewardship } \\
\hline Cumulative duration of use $[\mathrm{d}]^{\star *}$ & $14.3(6.8-23.9)$ & $11.2(2.8-27.3)$ & $14.7(9.5-22.3)$ & 0.560 \\
\hline \multicolumn{5}{|c|}{ Outcome parameters } \\
\hline Mortality at $28 \mathrm{~d}$ & $8(30.8 \%)$ & $5(41.7 \%)$ & $3(21.4 \%)$ & 0.246 \\
\hline Mortality at $90 \mathrm{~d}$ & $12(46.2 \%)$ & $5(41.7 \%)$ & $7(50 \%)$ & 0.532 \\
\hline Hospital length of stay [d] ${ }^{* *}$ & $34(11-55)$ & $14(11-55)$ & $41(11-54)$ & 0.631 \\
\hline ICU length of stay $[\mathrm{d}]^{* * *}$ & $20(6-50)$ & $14(6-49)$ & $26(7-53)$ & 0.705 \\
\hline Mechanical ventilation $[\mathrm{h}]^{* * *}$ & $245(51-718)$ & $85(9-286)$ & $469(151-884)$ & $0.036^{*}$ \\
\hline Need for tracheostomy & $15(57.7 \%)$ & $4(33.3 \%)$ & $11(78.6 \%)$ & $0.026^{*}$ \\
\hline \multicolumn{5}{|c|}{ Course of the disease up to the start of treatment with inhaled tobramycin } \\
\hline Mechanical ventilation $[\mathrm{h}]^{\star \star \star \star}$ & --- & --- & $364(33-677)$ & --- \\
\hline
\end{tabular}

Legends: ARF: Acute Renal Failure; ALF: Acute Liver Failure; ARDS: Acute Respiratory Distress Syndrome; APACHE II: Acute Physiology and Chronic Health Evaluation II- Score; SOFA: Sepsis-Related Organ Failure Assessment Score; SAPS: Simplified Acute Physiology Score; CPIS: Clinical Pulmonary Infection Score; 3-MRGN: multi-resistant gram-Negative Bacteria with Resistance Against three Antibiotic Drug Classes; 4-MRGN: Multi-Resistant Gram-Negative Bacteria with Resistance Against Four Antibiotic Drug Classes. *median value for the observation period of 28 days; ** of antibiotics effective against Pseudomonas aeruginosa; ${ }^{* \star *}$ after first detection of Pseudomonas aeruginosa in respiratory tract secretion; ****between the start of mechanical ventilation and the start of treatment with tobramycin p.i.

Data expressed by number (percentage) or median with accompanying quartiles (Q1-Q3).

Table 2: Clinical and microbiological follow-up data as well as outcome parameters in patients suffering from VAP $(n=26)$.

In 14 of 18 surviving VAP patients (77.8\%), P. aeruginosa disappeared within the first 14 days after study inclusion, whereas three of the surviving 18 patients (16.7\%) remained positive after 28 days (two patients (18.2\%) without inhaled tobramycin and one patient $(14.3 \%)$ with inhaled tobramycin). The number of patients with ongoing VAP caused by $P$. aeruginosa did not differ significantly between the two study groups at the different time points. At 90 days after study inclusion, respiratory tract secretions were available for 3 surviving VAP patients and revealed a positive culture for $P$. aeruginosa in 1 case (1 patient (14.3\%) without inhaled tobramycin and 0 patients $(0 \%)$ with inhaled tobramycin). Multidrug resistant $P$. aeruginosa strains were found in $3(11.5 \%)$ VAP patients at the time of first occurrence. There was no resistance against four main antibiotic groups (4-MRGN) in any patient, whereas the bacterial strains of three patients (11.5\%) were resistant against three main antibiotic groups (3-MRGN). There was no resistance of any bacterial strain against tobramycin at the time of first occurrence (Table 1) (Electronic Supplementary Material 3). Within the 28-day observation period, 4-MRGN or 3-MRGN $P$. aeruginosa could be observed in $1(3.8 \%)$ and $3(11.5 \%)$ patients respectively. In $1(3.8 \%)$ VAP patient treated with inhaled tobramycin, $P$. aeruginosa developed resistance against tobramycin within the 28-day observation period. In contrast, there was no tobramycin resistance in patients suffering from VAP due to $P$. aeruginosa not being treated with inhaled tobramycin within this timeframe (Table 2). Although there was only a limited number of respiratory secretions at 90 days after study inclusion, the use of inhaled tobramycin did not result in the occurrence of tobramycin resistant $P$. aeruginosa strains in VAP patients. The same holds true for VAP patients not treated with inhaled tobramycin, although there was one $P$. aeruginosa strain fulfilling 4-MRGN criteria (but without being tobramycin resistant) according to the German Society for Hygiene and Microbiology. Besides, in bacterial organisms other than $P$. aeruginosa, no tobramycin resistance could be observed throughout the 28-day and 90-day observation period respectively.

A detailed summary of all other relevant microbiological findings in respiratory tract secretions (besides $P$. aeruginosa) of patients suffering from VAP as well as a detailed description of the ensuing antibiotic treatment regimens during the 28-day observation period is presented in Electronic Supplementary Material 4. In brief, meropenem (57.7\%) and piperacillin/tazobactam (50.0\%) were applied most frequently in VAP patients based on local resistance patterns. Focusing on intravenously administered antibiotic drugs effective against $P$. aeruginosa, the cumulative days of therapy with these drugs did not differ significantly between the two study groups, with a median of $14.7 \mathrm{~d}$ (9.5-22.3 d) in VAP patients treated with inhaled tobramycin and $11.2 \mathrm{~d}(2.8-27.3 \mathrm{~d})$ in VAP patients treated with i.v. antibiotics only (Table 2). Moreover, it seems particularly important that the pulmonary microbiome did not differ significantly between patients treated with or without inhaled tobramycin within the 28day as well as 90-day observation period (Electronic Supplementary Material 4). In another subgroup analysis, patients were divided into 
Citation: Decker SO, Riebold da Costa R, Schmidt K, Uhle F, Lichtenstern C, et al. (2018) New Approaches to Anti-infective Treatment of VentilatorAssociated Respiratory Infections caused by Pseudomonas aeruginosa. J Med Microb Diagn 7: 270. doi:10.4172/2161-0703.1000270

Page 5 of 9

three groups (based on the three most frequently used intravenously administered antibiotics effective against $P$. aeruginosa: 1.) meropenem $(\mathrm{n}=15), 2$.) piperacillin/tazobactam $(\mathrm{n}=14), 3$.) ciprofloxacin $(\mathrm{n}=10))$ and afterwards analyzed for differences in the pulmonary microbiome. However, a significant difference between these three subgroups could not be observed (data not shown)

\section{Subgroup of patients with VAT $(n=55)$}

Patients fulfilling VAT-criteria $(n=55)$ were shown to be hospitalized for a median of $19.0 \mathrm{~d}$ (13.0-40.5 d) before study inclusion and required invasive ventilation for a duration of $238 \mathrm{~h}$ (73-454 h). Apart from oncologic diseases, no significant differences could be observed concerning baseline clinical data. As assessed by a significantly prolonged need for invasive ventilation prior to study inclusion, inhaled tobramycin was used in terms of a rescue therapy in patients suffering from VAT. No significant differences could be observed with regard to the prevalence of $P$. aeruginos a strains fulfilling 3- or 4-MRGN criteria at study inclusion (Table 3).

Within the 28-day observation period, VAT patients treated with inhaled tobramycin were shown to suffer from a more severe disease state, as assessed by significantly increased median SAPS- and CPISscores. In parallel, ALF occurred more frequently in VAT patients treated with inhaled tobramycin, whereas the incidence of ARF was comparable between the two subgroups. Moreover, the detection of $P$. aeruginosa strains fulfilling 3- or 4-MRGN criteria or presenting with tobramycin resistance, did not differ significantly between patients treated or not treated with inhaled tobramycin (Table 4). Regarding tobramycin-related side effects, none of the participating patients suffered from bronchoconstrictive episodes or coughing attacks during the inhalation procedure.
A detailed summary of all other relevant microbiological findings in respiratory tract secretions (besides $P$. aeruginosa) of patients suffering from VAT as well as a detailed description of the ensuing antibiotic treatment regimens during the 28 -day observation period is presented in Electronic Supplementary Material 5. In brief, ciprofloxacin (49.1\%), and ceftazidime (45.5\%) were applied most frequently in these patients. Focusing on intravenously administered antibiotic drugs potentially effective against $P$. aeruginosa, the cumulative days under therapy with these drugs did not differ significantly between the two study groups, with a median of $13.0 \mathrm{~d}$ (6.9-20.1 d) in VAT patients treated with inhaled tobramycin and $14.1 \mathrm{~d}(8.2-24.6 \mathrm{~d})$ in VAT patients only treated with i.v. antibiotics (Table 4).

At 90 days after study inclusion, respiratory tract secretions were available in 5 surviving VAT patients and revealed a positive culture for $P$. aeruginosa in 4 cases ( 0 patients $(0 \%)$ without inhaled tobramycin and 4 patients $(28.6 \%)$ with inhaled tobramycin). P. aeruginosa strains in these four VAT patients treated with inhaled tobramycin were either shown to be multi-sensible $(n=1 ; 7.1 \%)$ or fulfilled $3-\&$ 4-MRGN criteria in two $(14.3 \%)$ and one $(7.1 \%)$ case respectively. Most interestingly, one of these 3-MRGN $P$. aeruginosa strains was shown to be tobramycin resistant. Besides, in bacterial organisms other than $P$. aeruginosa, no tobramycin resistance could be observed throughout the 28-day and 90-day observation period respectively. Moreover, it seems particularly important, that the rest of the pulmonary microbiome did not differ significantly between patients treated with or without inhaled tobramycin within the 28-day as well as 90-day follow up (Electronic Supplementary Material 5). In another subgroup analysis, patients were divided into three groups (based on the three most frequently used intravenously administered antibiotics effective against $P$. aeruginosa: 1$)$ ciprofloxacin $(\mathrm{n}=27), 2)$ ceftazidime

\begin{tabular}{|c|c|c|c|c|}
\hline Variables & VAT patients $(n=55)$ & without tobramycin p.i. $(n=24)$ & with tobramycin p.i. $(n=31)$ & p-value \\
\hline \multicolumn{5}{|l|}{ Demographic data } \\
\hline Gender male [n] & $40(72.7 \%)$ & $16(66.7 \%)$ & $24(77.4 \%)$ & 0.279 \\
\hline Age (years) & $63(53-68)$ & $64.5(49.8-68)$ & $62(57.5-66)$ & 0.727 \\
\hline \multicolumn{5}{|l|}{ Known illnesses } \\
\hline Diabetes mellitus & $18(32.7 \%)$ & $11(45.8 \%)$ & $7(22.6 \%)$ & 0.063 \\
\hline Arterial hypertension & $36(65.5 \%)$ & $16(66.7 \%)$ & $20(64.5 \%)$ & 0.549 \\
\hline Coronary heart disease & $15(27.3 \%)$ & $8(33.3 \%)$ & $7(22.6 \%)$ & 0.279 \\
\hline Chronic obstructive lung disease & $9(16.4 \%)$ & $2(8.3 \%)$ & $7(22.6 \%)$ & 0.147 \\
\hline Renal insufficiency & $23(41.8 \%)$ & $10(41.7 \%)$ & $13(41.9 \%)$ & 0.601 \\
\hline Hepatic insufficiency & $21(38.2 \%)$ & $7(29.2 \%)$ & $14(45.2 \%)$ & 0.176 \\
\hline Oncologic disease & $17(30.9 \%)$ & $2(8.3 \%)$ & $15(48.4 \%)$ & $0.041^{*}$ \\
\hline Transplantation & $13(23.6 \%)$ & $6(25 \%)$ & $7(22.6 \%)$ & 0.541 \\
\hline \multicolumn{5}{|c|}{ Baseline clinical data* } \\
\hline Hospital length of stay [d] & $19(13-40.5)$ & $17(10-34)$ & $31(17-43.5)$ & 0.075 \\
\hline ICU length of stay [d] & $18(10-38.5)$ & $16(9-33)$ & $25(14.5-40)$ & 0.114 \\
\hline Mechanical ventilation [h] & $238(73-454)$ & $139(45-316)$ & $315(110-543)$ & $0.027^{*}$ \\
\hline APACHE II & $14(11-19)$ & $14(12-20)$ & $14(10-18)$ & 0.345 \\
\hline SOFA & $8(4-12)$ & $8(5-11)$ & $8(4-12)$ & 0.912 \\
\hline SAPS & $52(36-63)$ & $51(31-60)$ & $54(45-63)$ & 0.300 \\
\hline $\mathrm{paO} 2 / \mathrm{FiO}_{2}$ & $285(210-350)$ & $295(198-343)$ & $285(210-350)$ & 0.734 \\
\hline \multicolumn{5}{|c|}{ Baseline microbiological data* } \\
\hline Pseudomonas 3-MRGN & $25(45.5 \%)$ & $12(50 \%)$ & $13(41.9 \%)$ & 0.415 \\
\hline Pseudomonas 4-MRGN & $0(0 \%)$ & $0(0 \%)$ & $0(0 \%)$ & - \\
\hline Tobramycin-resistance & $0(0 \%)$ & $0(0 \%)$ & $0(0 \%)$ & - \\
\hline
\end{tabular}

Legends: APACHE II: Acute Physiology and Chronic Health Evaluation II - Score; SOFA: Sepsis-related Organ Failure Assessment score; SAPS: Simplified Acute Physiology Score; 3-MRGN: multi-resistant gram-negative bacteria with resistance against three main antibiotic drug classes; 4-MRGN: multi-resistant gram-negative bacteria with resistance against four main antibiotic drug classes; *prior to first detection of Pseudomonas aeruginosa in respiratory tract secretion Data expressed by number (percentage) or median with accompanying quartiles (Q1-Q3).

Table 3: Demographic and baseline clinical data of patients suffering from VAT $(n=55)$. 
Citation: Decker SO, Riebold da Costa R, Schmidt K, Uhle F, Lichtenstern C, et al. (2018) New Approaches to Anti-infective Treatment of VentilatorAssociated Respiratory Infections caused by Pseudomonas aeruginosa. J Med Microb Diagn 7: 270. doi:10.4172/2161-0703.1000270

Page 6 of 9

\begin{tabular}{|c|c|c|c|c|}
\hline Variables & VAT patients $(n=55)$ & without tobramycin p.i. $(n=24)$ & with tobramycin p.i. $(n=31)$ & p-value \\
\hline \multicolumn{5}{|c|}{ Organ failures up to day 28} \\
\hline ARF & $35(63.6 \%)$ & $14(58.3 \%)$ & $21(67.7 \%)$ & 0.330 \\
\hline ALF & $42(76.4 \%)$ & $14(58.3 \%)$ & $28(90.3 \%)$ & $0.036^{*}$ \\
\hline ARDS & $7(12.7 \%)$ & $3(12.5 \%)$ & $4(12.9 \%)$ & 0.508 \\
\hline \multicolumn{5}{|c|}{ Disease severity scores up to 28 days ${ }^{*}$} \\
\hline APACHE II & $10.4(7.1-14.6)$ & $9.9(6.4-14)$ & $11.8(8.1-14.6)$ & 0.203 \\
\hline SOFA & $5.1(2.5-7.9)$ & $3.7(2.6-7.2)$ & $6.1(2.6-8.3)$ & 0.231 \\
\hline SAPS & $37.7(22.5-45.1)$ & $29.1(20-41.4)$ & $41.1(29.6-47.1)$ & $0.030^{*}$ \\
\hline CPIS & $1.6(0.8-2.7)$ & $1.4(0.6-1.8)$ & $2.2(1-2.8)$ & $0.047^{*}$ \\
\hline \multicolumn{5}{|c|}{ Microbiological follow-up data within 28 days } \\
\hline Pseudomonas 3-MRGN & $13(23.6 \%)$ & $6(25 \%)$ & $7(22.5 \%)$ & 0.321 \\
\hline Pseudomonas 4-MRGN & $12(21.8 \%)$ & $6(25 \%)$ & $6(19.4 \%)$ & 0.546 \\
\hline Tobramycin-resistance & $8(14.5 \%)$ & $7(29.2 \%)$ & $1(3.2 \%)$ & 0.061 \\
\hline \multicolumn{5}{|c|}{ Final microbiological follow-up within 90 days } \\
\hline Pseudomonas 3-MRGN & $2(3.6 \%)$ & $0(0 \%)$ & $2(6.5 \%)$ & 0.222 \\
\hline Pseudomonas 4-MRGN & $1(1.8 \%)$ & $0(0 \%)$ & $1(3.2 \%)$ & 0.500 \\
\hline Tobramycin-resistance & $1(0 \%)$ & $0(0 \%)$ & $1(3.2 \%)$ & 0.500 \\
\hline \multicolumn{5}{|c|}{ Antibiotic stewardship } \\
\hline Cumulative duration of use $[\mathrm{d}]^{\star \star}$ & $13.3(7.8-22.3)$ & $14.1(8.2-24.6)$ & $13(6.9-20.1)$ & 0.734 \\
\hline \multicolumn{5}{|c|}{ Outcome parameters } \\
\hline Mortality at $28 \mathrm{~d}$ & $18(32.7 \%)$ & $7(29.2 \%)$ & $11(35.5 \%)$ & 0.521 \\
\hline Mortality at $90 \mathrm{~d}$ & $27(49.1 \%)$ & $10(41.7 \%)$ & $17(54.8 \%)$ & 0.243 \\
\hline Hospital length of stay $[d]^{\star * *}$ & $27(11-48.5)$ & $19(11-45)$ & $31(15-48.5)$ & 0.373 \\
\hline ICU length of stay $[\mathrm{d}]^{\star \star *}$ & $21(10-39)$ & $16(7-34)$ & $23(10-43)$ & 0.116 \\
\hline Mechanical ventilation $[\mathrm{h}]^{\star * *}$ & $204(31-474)$ & $48(0-301)$ & $303(87-602)$ & $0.010^{*}$ \\
\hline Need for tracheostomy & $35(63.7 \%)$ & $13(54.2 \%)$ & $22(71 \%)$ & 0.158 \\
\hline
\end{tabular}

Legends: ARF: Acute Renal Failure; ALF: Acute Liver Failure; ARDS: Acute Respiratory Distress Syndrome; APACHE II: Acute Physiology and Chronic Health Evaluation II - Score; SOFA: Sepsis-Related Organ Failure Assessment score; SAPS: Simplified Acute Physiology Score; CPIS: Clinical Pulmonary Infection Score; 3-MRGN: MultiResistant Gram-Negative Bacteria with Resistance against Three Antibiotic Drug Classes; 4-MRGN: Multi-Resistant Gram-Negative Bacteria with Resistance against Four Antibiotic Drug Classes. *median value for the observation period of 28 days; ${ }^{* *}$ of antibiotics effective against Pseudomonas aeruginosa; ${ }^{* *}$ after first detection of Pseudomonas aeruginosa in respiratory tract secretion;

Data expressed by number (percentage) or median with accompanying quartiles (Q1-Q3).

Table 4: Clinical and microbiological follow-up data as well as outcome parameters of patients suffering from VAT ( $n=55)$.

$(\mathrm{n}=25), 3)$ piperacillin/tazobactam $(\mathrm{n}=20))$ and afterwards analyzed for differences in the pulmonary microbiome. However, a significant difference between these three subgroups could not be observed (data not shown).

\section{Whole cohort of patients $(\mathrm{n}=\mathbf{8 1})$ suffering from ventilator- associated pulmonary infections}

Non-surprisingly, results of the whole cohort of patients suffering from ventilator-associated pulmonary infections are completely in line with the findings of the previously described subgroups of patients suffering from VAP or VAT: (1) Patients treated with inhaled tobramycin were shown to be more seriously ill as assessed by several disease severity scores and were hallmarked by (2) a longer need for mechanical ventilation as well as (3) a higher rate of tracheostomy following study inclusion. However, this might also be due to the fact that inhaled tobramycin was mostly used as a rescue therapy in cases of recurrent and/or persistent pulmonary infections caused by $P$. aeruginosa. Accordingly, hospital and ICU lengths of stay, as well as the duration of mechanical ventilation up to study inclusion tended to be increased in patients treated with inhaled tobramycin in comparison to patients not treated with inhaled tobramycin. Moreover, the use of inhaled tobramycin did not result in an increased risk for multiresistant $P$. aeruginosa strains at 28 days as well as 90 days following study inclusion (Electronic Supplementary Material 6). Quite the contrary, the rate of tobramycin-resistant $P$. aeruginosa strains was significantly increased in the subgroup of patients not treated with inhaled tobramycin $(n=7 ; 19.4 \%)$ in comparison to those patients treated with inhaled tobramycin $(n=2 ; 4.4 \%)$ at 28 days following the onset of the disease. In bacterial organisms other than $P$. aeruginosa, no tobramycin resistances could be observed throughout the 28-day and 90-day observation period respectively. In another subgroup analysis, patients were divided into four groups (based on the four most frequently used intravenously administered antibiotics effective against $P$. aeruginosa: 1$)$ ciprofloxacin $(\mathrm{n}=37), 2)$ ceftazidime $(\mathrm{n}=35), 3)$ piperacilin/tazobactam $(n=33), 4)$ meropenem $(n=33))$ and afterwards analyzed for differences in the pulmonary microbiome. However, in line with the results of the subgroups of patients with VAP or VAT, significant differences between these subgroups could not be observed (data not shown).

\section{Discussion}

In this retrospective analysis of critically ill patients suffering from recurrent and/or persisting VAP or VAT caused by $P$. aeruginosa, recent strategies for the anti-infective treatment as well as the risk profile of inhaled tobramycin were evaluated. Within this context, inhaled tobramycin was most frequently used for the augmentation of intravenously administered antibiotics and was shown to have a favorable risk profile.

VAP is a severe complication in ventilated patients as it is associated with the need for prolonged mechanical ventilation, prolonged ICU stay and an increased mortality [6]. Multidrug resistant strains play an important role in VAP $[7,23]$. Looking at the data from the European Centre for Disease Prevention and Control (ECDC) network, the 
Citation: Decker SO, Riebold da Costa R, Schmidt K, Uhle F, Lichtenstern C, et al. (2018) New Approaches to Anti-infective Treatment of VentilatorAssociated Respiratory Infections caused by Pseudomonas aeruginosa. J Med Microb Diagn 7: 270. doi:10.4172/2161-0703.1000270

Page 7 of 9

rates of multidrug resistant $P$. aeruginosa strains are increasing and therapeutic options are limited [24]. However, the first antibiotic shot should be a winner, since a delay in the initiation of an adequate anti-infective treatment is independently predictive for mortality in critically ill patients suffering from bacteremia with $P$. aeruginosa (due to a pulmonary focus in $77 \%$ of all cases) [25]. Accordingly, in severe infections caused by $P$. aeruginosa, there is some evidence for the use of an empiric combination therapy (e.g. cefepime + amikacin) instead of an empiric monotherapy (e.g. solely cefepime) [26]. These results are supported by another investigation by the group of Garnacho-Montero et al. performed in patients suffering from VAP due to $P$. aeruginosa. In these patients, the use of an initial combination therapy was superior to an empiric monotherapy with regard to clinical outcome measures (e.g. recurrent VAP, mortality, ICU mortality or in-hospital mortality), probably due to a significant reduction in the risk of an ineffective empiric antibiotic therapy in the initial phase of the disease [27]. As soon as the initial empiric combination therapy was deescalated towards a targeted antibiotic monotherapy, the continuation of a combination therapy did not provide any further advantages [27]. Therefore, the value of an initial empiric combination therapy seems to be primarily related to the reduction in the risk of inadequate initial treatment in patients at high risk of acquiring multidrug resistant strains (e.g. P. aeruginosa). Accordingly, in our investigation dealing with patients suffering from VAP due to $P$. aeruginosa, an i.v. combination therapy was performed in $35 \%$ (Group IV; $n=9$ ) of all patients, but was not associated with improvements in survival or better clinical outcome measures (e.g. disease severity scores, length of ICU stay, length of hospital stay, need for mechanical ventilation) in comparison to an initial empiric i.v. monotherapy (Group I; $n=12$ ). However, this finding should be interpreted cautiously due to the small cohort of patients included in the present investigation and does not offer a clear recommendation for action with regard to the preferable antibiotic treatment regime.

Due to potential negative side effects of an intravenous combination therapy (e.g. nephrotoxicity in the case of intravenously administered aminoglycosides $[28,29])$, the use of inhaled antibiotics may represent a desirable option for the augmentation of an antibiotic treatment in pulmonary infections due to its favorable risk-benefit profile. The advantages include the delivery of high local doses in combination with minimal systemic toxicity. The inhaled application of antibiotics is wellestablished in patients with cystic fibrosis [30-36]. However, the number of studies on the use of inhaled tobramycin in ventilated patients with VAP or VAT is limited, such that recent guidelines give only a weak recommendation for the additional use of inhaled antibiotics, especially in patients at high risk of acquiring multidrug resistant strains; this highlights the urgent need for more scientific research on this topic $[17,18]$. One interesting case-control study in oncologic patients suffering from VAP caused by gram-negative strains (e.g. P. aeruginosa in $69 \%$ of cases) was published by Ghannam et al. [37]. Within this investigation, the use of inhaled antibiotics (e.g. aminoglycosides as well as colistin) was superior to an intravenous treatment strategy with regard to several clinical and microbiological infection criteria (e.g. nephrotoxicity, resolution of clinical and microbiologic infections). Another retrospective investigation by Czosnowski et al. also supports the adjunctive use of inhaled tobramycin in VAP patients [38]. A comparable study to ours was performed by Arnold et al. [39]. The dose of $300 \mathrm{mg}$ tobramycin twice daily was similar, and the number of patients treated with inhaled tobramycin was also comparable. The need for mechanical ventilation was increased in the group of patients treated with inhaled tobramycin in both studies. Moreover, trends concerning the duration of ICU and hospital stay, as well as 28-day survival, were comparable in both studies. This might be due to the fact that patients receiving inhaled tobramycin were found to be sicker and suffered from recurrent and/or persistent VAP in both investigations as demonstrated by higher APACHE-II scores in the study by Arnold et al. and a prolonged need for mechanical ventilation and a higher rate of tracheostomy in our study. Additionally, in the study by Arnold et al., not all patients were positive for $P$. aeruginosa in the respiratory tract, since patients suffering from VAP caused by Acinetobacter baumanii were also included. Another study dealing with the use of inhaled antibiotics in critically ill ICU patients suffering from VAP caused by P. aeruginosa or Acinetobacter spp. was performed by Hallal et al. [16], comparing the use of inhaled tobramycin $(n=5)$ with intravenously administered tobramycin $(n=8)$ in a randomized, double-blind pilot study. This investigation was able to show that all patients treated with inhaled tobramycin had clinical resolution of VAP, whereas two intravenously treated patients were considered to suffer from treatment failures (deterioration of multiorgan dysfunction score, doubling of serum creatinine as a sign of nephrotoxicity). Although the design of the study by Hallal et al. is completely different to ours and a comparison of the results therefore have to be performed with caution, the following key aspect is supported throughout both investigations: the use of inhaled tobramycin does not lead to relevant systemic side effects (e.g. nephrotoxicity) and is therefore associated with a suitable risk profile. This is further supported by the results of Florescu et al. [40] and van Koningsbruggen-Rietschel et al. [41]. Both studies evaluated the effect of inhaled tobramycin in patients with cystic fibrosis and did not observe nephrotoxic side effects with this route of administration. However, the use of empiric monotherapy with inhaled tobramycin as described by Hallal et al. has only been performed in one patient within the present investigation in terms of individual treatment. This is in line with recent guidelines, which actually do not recommend monotherapy with inhaled antibiotics due to the lack of clear evidence $[17,18]$. Besides nephrotoxicity, the induction of bronchoconstriction or coughing attacks has been described as another potential side effect during the use of inhaled tobramycin $[33,42,43]$. However, none of the participating patients suffered from such episodes during the inhalation procedure, indicating that inhaled tobramycin is well-tolerated even in critically ill ICU patients with an irritated bronchial system. The induction of resistance mechanisms due to the use of inhaled tobramycin was also suspected to be a potential danger. As previously described by the EARS, multidrug resistant $P$. aeruginosa strains (3-MRGN/4-MRGN) are increasingly important [24]. Accordingly, $P$. aeruginosa strains of all patients within the presented investigation revealed a 3-MRGN resistance pattern in $34.6 \%(\mathrm{n}=28)$, whereas none of the strains appeared to be 4-MRGN prior to the onset of the disease. This resistance condition took a turn for the worse 28-days as well as 90-days later, since $P$. aeruginosa strains appeared to reveal a 4-MRGN resistance pattern much more frequently (at 28 days: $n=13$ (16\%); at 90 days: $n=2(2.5 \%)$ ), but without being attributable to the use of inhaled tobramycin. With regard to the development of tobramycin-resistant $P$. aeruginosa strains due to the use of inhaled tobramycin, the present investigation was able to dispel any existing reservations: in the whole cohort of patients suffering from respiratory infections caused by $P$. aeruginosa, the rate of tobramycin-resistant $P$. aeruginosa strains was significantly increased in the subgroup of patients not treated with inhaled tobramycin $(n=7 ; 19.4 \%)$ in comparison to those patients treated with inhaled tobramycin $(n=2 ; 4.4 \%)$ at 28 days following the onset of the disease. The same holds true for the rest of the pulmonary microbiome, since inhaled tobramycin did not lead to a significant change in the composition of the microbiome and none of the identified 
Citation: Decker SO, Riebold da Costa R, Schmidt K, Uhle F, Lichtenstern C, et al. (2018) New Approaches to Anti-infective Treatment of VentilatorAssociated Respiratory Infections caused by Pseudomonas aeruginosa. J Med Microb Diagn 7: 270. doi:10.4172/2161-0703.1000270

Page 8 of 9

bacterial species at 28 days as well as 90 days (others than P. aeruginosa) were shown to develop tobramycin-resistance. Accordingly, the use of inhaled tobramycin in patients suffering from respiratory infections caused by $P$. aeruginosa seems not to be associated with a deleterious effect on the pulmonary microbiome as well as an increased risk for the development of tobramycin resistant strains.

One further aim of the present study was to assess whether the use of inhaled tobramycin is associated with a reduced need for i.v. antibiotics. Unfortunately, this aim could not be achieved due to the following reasons: (1) Besides VAP or VAT, most of the patients suffered from a severe leading infection (e.g. in the abdominal cavity) requiring i.v. antibiotics. Therefore, the amount of i.v. antibiotics was more often affected by the clinical development of the leading infection than by the progression of VAP or VAT. (2) Methodological weaknesses of the retrospective design might have led to an underestimation of the advantages of a treatment regime with inhaled tobramycin. Therefore, a prospective reevaluation for the use of inhaled tobramycin in patients suffering from VAP or VAT is recommended. Moreover, it would be more than interesting to assess whether a systematic use of inhaled tobramycin in patients suffering from VAT might be able to avoid the conversion of VAT into VAP with all its potentially deleterious consequences.

With regard to limitations, the following aspects need to be discussed critically. The study design was retrospective and only a restricted number of patients were included. Moreover, the field of application for the use of inhaled tobramycin was not clearly defined, resulting in heterogeneous study groups. Whether inhaled tobramycin is able to prevent the conversion of VAT into VAP cannot be concluded by the present investigation and needs to be addressed in an additional prospective project.

\section{Conclusion}

Inhaled tobramycin seems to be a suitable approach with a favorable risk profile for the treatment of patients suffering from recurrent and/ or persistent VAP or VAT caused by P. aeruginosa. However, "hard" clinical benefits of this treatment regime need to be reevaluated in a large prospective investigation.

\section{Acknowledgements}

The study was carried out with financial resources of the Department of Anesthesiology at Heidelberg University Hospital, Germany.

\section{Authors' contributions}

SOD, AH, MAW and TBre participated in the design of the study and drafted the manuscript. RR carried out the data analysis. TBru was responsible for the biometric design and performed statistical analyses. KS, FU, CL, DS, AU and SH participated in the design of the study and critically revised the manuscript. All authors read and approved the final manuscript.

\section{Declaration of Conflicting Interests}

The authors declare that there is no conflict of interest.

\section{References}

1. Warrell DA (2003) The Oxford Textbook of Medicine. (4th edn), Oxford University Press, UK

2. Mandell GL (2004) Mandell's Principles and Practices of Infection Diseases. (6th edn), Churchill Livingstone, UK.

3. Kasper DL (2005) Harrison's Principles of Internal Medicine. (16th edn), The McGraw-Hill Companies, USA.

4. American Thoracic Society and Infectious Diseases Society of America (2005) Guidelines for the management of adults with hospital-acquired, ventilatorassociated, and healthcare-associated pneumonia. Am J Respir Crit Care Med 171: 388-416.
5. van der Kooi TI, de Boer AS, Mannien J, Wille JC, Beaumont MT, et al. (2007) Incidence and risk factors of device-associated infections and associated mortality at the intensive care in the Dutch surveillance system. Intensive Care Med 33: 271-278.

6. Nseir S, Favory R, Jozefowicz E, Decamps F, Dewavrin F, et al. (2008) Antimicrobial treatment for ventilator-associated tracheobronchitis: a randomized, controlled, multicenter study. Crit Care 12: R62.

7. Nair GB, Niederman MS (2015) Ventilator-associated pneumonia: present understanding and ongoing debates. Intensive Care Med 41: 34-48.

8. Resar R, Pronovost P, Haraden C, Simmonds T, Rainey T, et al. ( 2005) Using a bundle approach to improve ventilator care processes and reduce ventilatorassociated pneumonia. Jt Comm J Qual Patient Saf 3: 243-248.

9. Kollef $\mathrm{MH}$ (2001) Hospital-acquired pneumonia and de-escalation of antimicrobial treatment. Crit Care Med 29: 1473-1475.

10. Adair CG, Gorman SP, Byers LM, Jones DS, Feron B, et al. (2002) Eradication of endotracheal tube biofilm by nebulised gentamicin. Intensive Care Med 28: 426-431.

11. Levy J, Smith AL, Kenny MA, Ramsey B, Schoenknecht FD (1983) Bioactivity of gentamicin in purulent sputum from patients with cystic fibrosis or bronchiectasis: comparison with activity in serum. J Infect Dis 148: 1069-1076.

12. Marr AK, Overhage J, Bains M, Hancock RE (2007) The Lon protease of Pseudomonas aeruginosa is induced by aminoglycosides and is involved in biofilm formation and motility. Microbiology 153: 474-482.

13. Mendelman PM, Smith AL, Levy J, Weber A, Ramsey B, et al. (1985) Aminoglycoside penetration, inactivation, and efficacy in cystic fibrosis sputum. Am Rev Respir Dis 132: 761-765

14. Le J, Ashley ED, Neuhauser MM, Brown J, Gentry C, et al. (2010) Consensus summary of aerosolized antimicrobial agents: application of guideline criteria Insights from the Society of Infectious Diseases Pharmacists. Pharmacotherapy 30: 562-584.

15. Drobnic ME, Sune P, Montoro JB, Ferrer A, Orriols R (2005) Inhaled tobramycin in non-cystic fibrosis patients with bronchiectasis and chronic bronchial infection with $P$. aeruginosa. Ann Pharmacother 39: 39-44.

16. Hallal A, Cohn SM, Namias N, Habib F, Baracco G, et al. (2007) Aerosolized tobramycin in the treatment of ventilator-associated pneumonia: a pilot study. Surg Infect (Larchmt) 8: 73-82.

17. Dalhoff K, Abele-Horn M, Andreas S, Bauer T, von Baum H, et al. (2012) Epidemiology, diagnosis and treatment of adult patients with nosocomial pneumonia. S-3 Guideline of the German Society for Anaesthesiology and Intensive Care Medicine, the German Society for Infectious Diseases, the German Society for Hygiene and Microbiology, the German Respiratory Society and the Paul-Ehrlich-Society for Chemotherapy. Pneumologie 66: 707-765.

18. Kalil AC, Metersky ML, Klompas M, Muscedere J, Sweeney DA, et al. (2016) Management of adults with hospital-acquired and ventilator-associated pneumonia: 2016 Clinical Practice Guidelines by the Infectious Diseases Society of America and the American Thoracic Society. Clin Infect Dis 63: e61-e111.

19. Rello J, Sole-Lleonart C, Rouby JJ, Chastre J, Blot S, et al. (2017) Use of nebulized antimicrobials for the treatment of respiratory infections in invasively mechanically ventilated adults: A position paper from the European Society of Clinical Microbiology and Infectious Diseases. Clin Microbiol Infect 23: 629-639.

20. Agrafiotis M, Siempos I, Falagas ME (2010) Frequency, prevention, outcome and treatment of ventilator-associated tracheobronchitis: systematic review and meta-analysis. Respir Med 104: 325-336.

21. Craven DE (2008) Ventilator-associated tracheobronchitis (VAT): Questions answers, and a new paradigm?. Crit Care 12: 157

22. Horan TC, Andrus M, Dudeck MA (2008) CDC/NHSN surveillance definition of health care-associated infection and criteria for specific types of infections in the acute care setting. Am J Infect Control 36: 309-332.

23. Martin-Loeches I, Deja M, Koulenti D, Dimopoulos G, Marsh B, et al. (2013) Potentially resistant microorganisms in intubated patients with hospital-acquired pneumonia: The interaction of ecology, shock and risk factors. Intensive Care Med 39: 672-681.

24. European Center for Disease, Prevention and Control: "Proportion of Carbapenems Resistant $(R+I)$ Pseudomonas aeruginosa Isolates in Participating Countries in 2014," 2015 
Citation: Decker SO, Riebold da Costa R, Schmidt K, Uhle F, Lichtenstern C, et al. (2018) New Approaches to Anti-infective Treatment of VentilatorAssociated Respiratory Infections caused by Pseudomonas aeruginosa. J Med Microb Diagn 7: 270. doi:10.4172/2161-0703.1000270

25. Kang Cl, Kim SH, Kim HB, Park SW, Choe YJ, et al. ( 2003) Pseudomonas aeruginosa bacteremia: Risk factors for mortality and influence of delayed receipt of effective antimicrobial therapy on clinical outcome. Clin Infect Dis 37: 745-751

26. Chaudhary M, Shrivastava SM, Varughese L, Sehgal R (2008) Efficacy and safety evaluation of fixed dose combination of cefepime and amikacin in comparison with cefepime alone in treatment of nosocomial pneumonia patients. Curr Clin Pharmacol 3: 118-122.

27. Garnacho-Montero J, Sa-Borges M, Sole-Violan J, Barcenilla F, EscorescaOrtega A, et al. (2007) Optimal management therapy for Pseudomonas aeruginosa ventilator-associated pneumonia: An observational, multicenter study comparing monotherapy with combination antibiotic therapy. Crit Care Med 35: 1888-1895.

28. Martinez-Salgado C, Lopez-Hernandez FJ, Lopez-Novoa JM (2007) Glomerular nephrotoxicity of aminoglycosides. Toxicol Appl Pharmacol 223: 86-98.

29. Sack K, Wilhelm J (1973) Comparative studies on nephrotoxicity of aminoglycosides: gentamicin and tobramycin. Verh Dtsch Ges Inn Med 79: 676-678.

30. Doring G, Flume P, Heijerman H, Elborn JS, Consensus Study Group (2012) Treatment of lung infection in patients with cystic fibrosis: current and future strategies. J Cyst Fibros 11: 461-479.

31. Gibson RL, Emerson J, McNamara S, Burns JL, Rosenfeld M, et al. (2003) Significant microbiological effect of inhaled tobramycin in young children with cystic fibrosis. Am J Respir Crit Care Med 167: 841-849.

32. Hodson ME, Gallagher CG, Govan JR (2002) A randomised clinical trial of nebulised tobramycin or colistin in cystic fibrosis. Eur Respir J 20: 658-664.

33. Pai VB, Nahata MC (2001) Efficacy and safety of aerosolized tobramycin in cystic fibrosis. Pediatr Pulmonol 32: 314-327.

34. Ramsey BW, Dorkin HL, Eisenberg JD, Gibson RL, Harwood IR, et al. (1993) Efficacy of aerosolized tobramycin in patients with cystic fibrosis. N Engl J Med 328: $1740-1746$
35. Ramsey BW, Pepe MS, Quan JM, Otto KL, Montgomery AB, Jet al. (1999) Intermittent administration of inhaled tobramycin in patients with cystic fibrosis Cystic Fibrosis Inhaled Tobramycin Study Group. N Engl J Med 340: 23-30.

36. Sawicki GS, Signorovitch JE, Zhang J, Latremouille-Viau D, von Wartburg M, et al. (2012) Reduced mortality in cystic fibrosis patients treated with tobramycin inhalation solution. Pediatr Pulmonol 47: 44-52.

37. Ghannam DE, Rodriguez GH, Raad II, Safdar A (2009) Inhaled aminoglycosides in cancer patients with ventilator-associated Gram-negative bacteria pneumonia: Safety and feasibility in the era of escalating drug resistance. Eur J Clin Microbiol Infect Dis 28: 253-259.

38. Czosnowski QA, Wood GC, Magnotti LJ, Croce MA, Swanson JM, et al (2009) Adjunctive aerosolized antibiotics for treatment of ventilator-associated pneumonia. Pharmacotherapy 29:1054-1060.

39. Arnold HM, Sawyer AM, Kollef MH (2012) Use of adjunctive aerosolized antimicrobial therapy in the treatment of Pseudomonas aeruginosa and Acinetobacter baumannii ventilator-associated pneumonia. Respir Care 57 1226-1233.

40. Florescu MC, Lyden E, Murphy PJ, Florescu DF, Fillaus J (2012) Long-term effect of chronic intravenous and inhaled nephrotoxic antibiotic treatment on the renal function of patients with cystic fibrosis. Hemodial Int 16: 414-419.

41. van Koningsbruggen-Rietschel S, Heuer HE, Merkel N, Posselt HG, Staab D et al. (2016) Pharmacokinetics and safety of an 8 week continuous treatment with once-daily versus twice-daily inhalation of tobramycin in cystic fibrosis patients. J Antimicrob Chemother 71: 711-717.

42. Sexauer WP, Fiel SB (2003) Aerosolized antibiotics in cystic fibrosis. Semin Respir Crit Care Med 24: 717-726.

43. Mattner F, Bange FC, Meyer E, Seifert H, Wichelhaus TA, et al. (2012) Preventing the spread of multidrug resistant gram-negative pathogens: recommendations of an expert panel of the German Society For Hygiene and Microbiology. Dtsch Arztebl Int 109: 39-45. 